\title{
Frailty: Its Scope and Implications for Geriatricians
}

\author{
Chang Won Won \\ Department of Family Medicine, College of Medicine, Kyung Hee University, Seoul, Korea
}

With the aging of population, more older adults become frail and many become dependent or bedridden. Frailty is defined as a status of vulnerability to endogenous and exogenous stressors that increase the risk of negative health-related outcomes. Frailty is usually caused by the interaction between progressive age-related decline in physiologic systems and chronic diseases, leading to decreased functional reserve capacities. ${ }^{1)}$ Frailty is generally considered a transition between successful aging and disability. ${ }^{2)}$

\section{What is the Scope of Managing Frailty for Geriatricians?}

Disease to function-oriented focus: Frailty is a status of decreased functional reserve capacities. ${ }^{1)}$ Thus, frailty does not fit into the traditional medical hierarchy of 'health and disease'. Addressing frailty means a shift away from the disease-centered paradigm toward a focus on function. ${ }^{3)}$

Comprehensive approach: Frailty includes social and psychological components in addition to physical dysfunction. ${ }^{4)} \mathrm{A}$ longitudinal study showed that social frailty, defined as living alone, lack of social contacts, and lack of social support, was associated with the use of nursing care and contact numbers of health care professionals. ${ }^{5)}$ Cognitive dysfunction predicts physical frailty; moreover, physical frailty and cognitive impairment affect each other, resulting in worse outcomes. ${ }^{6}$ Therefore, screening for frailty requires comprehensive geriatric assessment. Hearing impairment and visual disability are also risk factors for frailty. ${ }^{7}$

Multimorbid, complex status: Frailty is frequently associated with a multimorbid status. In the Cardiovascular Health Study, $9.7 \%$ of older adults with multiple morbidities were frail, while $67.7 \%$ of frail older adults had multiple morbidities. ${ }^{8)}$ To address frailty, a 'complex systems' approach is needed to address multidimensional processes and 'complex relation of biological and non-biological factors' on which frailty is based. ${ }^{3)}$

Implications for chronic diseases: Frailty is an important con- sideration for geriatricians in the clinical setting, particularly with respect to treatment goals for chronic disease in older patients. ${ }^{9)}$ For example, guidelines from the European Society of Hypertension and the European Society of Cardiology recommend leaving decisions regarding antihypertensive therapy in frail older patients to the treating physician and considering treatment based on monitoring of the clinical effects of treatment and individual tolerability. ${ }^{10)}$ The American Geriatrics Society consensus panels recommend glycated hemoglobin levels between $7.5 \%$ and $8.0 \%$, particularly in individuals aged $>80$ years who are at a high risk for frailty, comorbid conditions, and polypharmacy. ${ }^{11)}$ The decision to treat primary hypercholesterolemia with statins in Older adults aged 80 or more must be individualized and frailty status must be considered to decide on it as frailty may exacerbate adverse effects of statins. ${ }^{12)}$

\section{Why is Frailty Important to Geriatricians?}

The management of frailty is the area in which the art of geriatrics is best practiced. Frailty has replaced the traditional concept of 'chronological age' with the more accurate and individually tailored 'biological age. ${ }^{1)}$ In other words, frailty shed light on individualized care. Frail older adults are vulnerable and complicated, with care needs requiring skill and experience.

For this reason, frailty is a giant geriatric syndrome important to geriatricians; in this domain, the real value of geriatrics is shining brightly. Geriatricians differ from other specialists by providing specialized care for frail older adults. Geriatricians should champion the care of complex, vulnerable, and complex frail older adults.

\section{Research and Implications}

The Korean Frailty and Aging Cohort Study (KFACS), funded by the Ministry of Health, has been ongoing since December 2015. The KFACS is a multicenter, longitudinal study, with a baseline 
survey conducted in 2016-2017 and a 2-year follow-up survey underway. The KFACS aims to identify risk factors for adverse outcomes associated with frailty and preventative measures in community-dwelling older adults.

The final goal of the research is to increase knowledge for the diagnosis and management of frailty for implementation in clinical practice to reduce disability and dependency of frail older adults. KFACS data revealed discrepancies in the prevalence of frailty scales. ${ }^{13)}$ Additionally, the risk of frailty was associated with limited contact with friends, ${ }^{14)}$ anorexia, ${ }^{15)}$ long sleep latency or long sleeping duration, ${ }^{16)}$ high sodium intake, ${ }^{17)}$ and low self-rating of health. ${ }^{18)}$ The KFACS dataset and laboratory findings are available to extramural researchers. ${ }^{19)}$

\section{CONFLICT OF INTEREST DISCLOSURES}

The author claims no conflicts of interest.

\section{ACKNOWLEDGEMENTS}

This research was supported by a grant from the Korea Health Technology R\&D Project through the Korean Health Industry Development Institute, funded by the Ministry of Health and Welfare of the Republic of Korea (No. HI15C3153).

\section{REFERENCES}

1. Cesari M, Prince M, Thiyagarajan JA, De Carvalho IA, Bernabei R, Chan P, et al. Frailty: an emerging public health priority. J Am Med Dir Assoc 2016;17:188-92.

2. Lang PO, Michel JP, Zekry D. Frailty syndrome: a transitional state in a dynamic process. Gerontology 2009;55:539-49.

3. Junius-Walker U, Onder G, Soleymani D, Wiese B, Albaina O, Bernabei R, et al. The essence of frailty: a systematic review and qualitative synthesis on frailty concepts and definitions. Eur J Intern Med 2018;56:3-10.

4. Abellan van Kan G, Rolland Y, Bergman H, Morley JE, Kritchevsky SB, Vellas B. The I.A.N.A Task Force on frailty assessment of older people in clinical practice. J Nutr Health Aging 2008; 12:29-37.

5. Gobbens RJ, van Assen MA, Luijkx KG, Schols JM. The predictive validity of the Tilburg Frailty Indicator: disability, health care utilization, and quality of life in a population at risk. Gerontologist 2012;52:619-31.

6. Won CW, Lee Y, Kim S, Yoo J, Kim M, Ng TP, et al. Modified criteria for diagnosing "Cognitive Frailty". Psychiatry Investig 2018;15:839-42.
7. Yoo M, Kim S, Kim BS, Yoo J, Lee S, Jang HC, et al. Moderate hearing loss is related with social frailty in a community-dwelling older adults: the Korean Frailty and Aging Cohort Study (KFACS). Arch Gerontol Geriatr 2019;83:126-30.

8. Fried LP, Tangen CM, Walston J, Newman AB, Hirsch C, Gottdiener J, et al. Frailty in older adults: evidence for a phenotype. J Gerontol A Biol Sci Med Sci 2001;56:M146-56.

9. Won CW, Kim S. Use of frailty in deciding clinical treatment goals for chronic disease in elderly patients in the community. J Am Med Dir Assoc 2016;17:967-9.

10. Mancia G, Fagard R, Narkiewicz K, Redon J, Zanchetti A, Bohm M, et al. 2013 ESH/ESC guidelines for the management of arterial hypertension: the Task Force for the Management of Arterial Hypertension of the European Society of Hypertension (ESH) and of the European Society of Cardiology (ESC). Eur Heart J 2013;34:2159-219.

11. Brown AF, Mangione CM, Saliba D, Sarkisian CA; California Healthcare Foundation/American Geriatrics Society Panel on Improving Care for Elders with Diabetes. Guidelines for improving the care of the older person with diabetes mellitus. J Am Geriatr Soc 2003;51(5 Suppl Guidelines):S265-80.

12. Strandberg TE, Kolehmainen L, Vuorio A. Evaluation and treatment of older patients with hypercholesterolemia: a clinical review. JAMA 2014;312:1136-44.

13. Kim KJ, Shin J, Choi J, Won CW. Discrepancies in the prevalence of known frailty scales: Korean Frailty and Aging Cohort Study. Ann Geriatr Med Res 2018;22:137-44.

14. Chon D, Lee Y, Kim J, Lee KE. The association between frequency of social contact and frailty in older people: Korean Frailty and Aging Cohort Study (KFACS). J Korean Med Sci 2018;33:e332.

15. Kim J, Lee Y, Won CW, Lee KE, Chon D. Nutritional status and frailty in community-dwelling older Korean adults: the Korean Frailty and Aging Cohort Study. J Nutr Health Aging 2018;22: 774-8.

16. Kang I, Kim S, Kim BS, Yoo J, Kim M, Won CW. Sleep latency in men and sleep duration in women can be frailty markers in community-dwelling older adults: the Korean Frailty and Aging Cohort Study (KFACS). J Nutr Health Aging 2019;23:63-7.

17. Kim S, Kim M, Min J, Yoo J, Kim M, Kang J, et al. How much intake of sodium is good for frailty?: the Korean Frailty and $\mathrm{Ag}$ ing Cohort Study (KFACS). J Nutr Health Aging 2019;23:5038.

18. Kim S, Won CW, Kim BS, Kim S, Yoo J, Byun S, et al. EuroQol Visual Analogue Scale (EQ-VAS) as a predicting tool for frailty in older Korean adults: the Korean Frailty an Aging Cohort Study (KFACS). J Nutr Health Aging 2018;22:1275-80. 
19. Won CW, Lee Y, Choi J, Kim KW, Park Y, Park H, et al. Starting construction of frailty cohort for elderly and intervention study. Ann Geriatr Med Res 2016;20:114-7.
Corresponding Author: Chang Won Won, $\mathrm{MD}, \mathrm{PhD}$

Department of Family Medicine, College of Medicine, Kyung Hee University, 23 Kyungheedae-ro, Dongdaemun-gu, Seoul 02447, Korea

E-mail: chunwon@khmc.or.kr

ORCID: https://orcid.org/0000-0002-6429-4461

Received: September 2, 2019; Accepted: September 4, 2019 